\title{
A NEW THEORY OF MEASUREMENT: A STUDY IN THE LOGIC OF MATHEMATICS
}

\author{
By Norbert Wiener.
}

[Read November 13th, 1919.]

\section{Introduction.}

It is a deeply rooted popular idea that mathematics is but another name for measurement. Notwithstanding the fact that the existence of such non-metrical branches of mathematics as projective and descriptive geometry, the theory of groups, the algebra of logic, \&c., prove this notion false, it is nevertheless true that the applications of mathematics have, up to the present time, been, almost without exception, applications of measurement. The natural sciences, in so far as they have been regarded as at all amenable to a mathematical treatment, have reduced themselves to the correlation of different ranges of measurement-of space, $\operatorname{time}$, and mass, in the case of physics, of intensity of stimulus and intensity of sensory experience, in the case of psychophysics, and so on. Now, things do not, in general, run around with their measures stamped on them like the capacity of a freight-car: it requires a certain amount of investigation to discover what their measures are. It is, then, a necessary preliminary to the most complete scientific work that we should possess an analysis of the process through which we go in measuring the magnitude of a thing.

Now, a very beautiful theory of measurement has been developed in the third volume of the Principia Mathematica of A. N. Whitehead and B. Russell. It depends on the consideration of "vector-families"; that is, of sets of vectors such that it is possible to go from any point of the field ordered by the vectors to one and only one point along any given vector. These vectors correspond to definite increments of the measures of the terms in the common field of the vectors: thus, in the "vector-family" representing distances to the right along a given line, from a given point, an increment of the distance of this given point from a point to the extent of one inch, or of two inches, \&c., is a vector. Since it is always possible to leave a point by any vector one pleases, our system of measurement 
must contain magnitudes larger by any desired amount than any given magnitude. As a consequence, measurement by "vector-families" breaks down when we have to deal with ranges of quantities that are essentially limited. As the authors of the Principia say of their theory, "We exclude magnitudes which have a definite maximum, unless they are circular, like the angles at a point, or the distances on an elliptic straight line."* The authors of the Principia justify this omission by the further statement, "But, except when they are circular, such magnitudes are of little importance." This is, in general, true, but there is one exception which is well worth considering. Perhaps the least satisfactory and most discussed portion of the unsatisfactory and much discussed theory of measurement is that which deals with the measurement of the intensities and qualities of sense-data. ${ }^{+}$Now, the intensities and qualities of sense-data are not susceptible to increments of arbitrary magnitude. There is no degree of loudness as much greater than that of a foghorn at close range as the loudness of this is greater than that of the ticking of a watch at the distance of ten feet. There is no note as much higher than that of the cricket as the latter is higher than the lowing of an ox. There is no object as much more intensely red than a drop of blood in the sunlight as the latter is than a piece of grey flannel. Nevertheless, we often do speak of the measure of the loudness or pitch of a note or the intensity of a colour, and if Weber's law is to have any meaning in any other form than that in which it refers to "just noticeable" differences in intensity or quality, ; we must be able to establish some intrinsic criterion whereby we can determine the ratio of one difference in sensation quality or intensity to another, which will not presuppose that such an interval can be increased by any numerical factor whatever.

The first steps which are essential to the measurement of sensor? qualities and intensities are the determination of the fundamental experience by means of which this measurement is to be performed, and the derivation from this crude, uncouth experience of functions which will have certain comparatively neat properties, and which hence will form a mere convenient starting point for the process of measurement proper. Our measurement of sensation-intensities obviously has its origin in the consideration of intensity-intervals between sensations : that is sufficientl?

* Vol. 3, p. 340, lines 1-4.

† Except in one respect, which we shall indicate later, the theories of qualities and intensities coincide.

$\ddagger$ Cf. "Studies in Synthetic Logic," Proc. Camb. Pkil. Soc., Vol. 18, Part 1, pp. 24-28, $\$ \$ 7,8$, by Norbert Wiener. 
indicated by the fact that our measurement of a sensation-intensity always reduces itself, sooner or later, to the determination of its ratio to some standard intensity, while such a proposition as " $x$ is twice as intense as $y$," is simply a paraphrase for some such statement as "The interval of intensity between $x$ and $y$ equals that between $y$ and some sensation of zero intensity." The fundamental experience for which we are searching is not, however, of the form "The interval between $x$ and $y$ equals that between $u$ and $v . "$ Not every two intervals which seem equal are equal : two intervals may only be subliminally different. Nevertheless, our only direct method of determining with what intervals an interval is equal lies, as we shall show, in the determination, first, of with what intervals it seems equal. But even the seeming equality of two intervals is not quite what we want: two intervals seem equal, as far as we are concerned, when and only when neither seems greater than the other. We shall take, then, as the relation which forms the basis of the measurement of sensation-intensities, "The interval between $x$ and $y$ seems less than that between $u$ and $v$," where intervals are regarded as possessing signs, ascending intervals being regarded as positive, and "less than" is to be interpreted as "algebraically less than." In taking this as our primitive experience, we do not mean to assert-in fact we should categorically deny-that this relation is given as such in our experience, and that no further analysis of it is possible: what we do assert is that it represents a much more minute analysis of the basis of our measurements of sensation-intensities than any yet given, and forms a convenient starting point for a theory of sensation-intensities.

Although our initial relation enables us to give a sort of order to all sensation-intervals which seem greater or less than other intervals in some definite way (i.e. as loudness-intervals or as brightness-intervals, \&c.), we shall, in our subsequent work, limit its range of application to positive supraliminal intervals, or intervals which seem greater than some interval between a thing and itself. We do this, because zero-intervals have certain properties which interfere with our subsequent theory. Later on, we shall use as our criterion of the genuine equality of two intervals the fact that all the intervals which are indistinguishable from (i.e. neither noticeably greater than nor noticeably less than) either are indistinguishable from the other. It will be possible, therefore, for us to say that two intervals may be indistinguishable in magnitude, yet that one is greater than the other. Let the interval between $x$ and $y$ and the interval between $x$ and $z$ be a pair of intervals of this sort. Then $y$ must be indistinguishable from $z$ by direct comparison, yet we must be able to say that $y$ and $z$ are not really of the same intensity, and hence that the interval 
between $y$ and $z$, though indistinguishable from the zero-interval, is not of measure zero, or else it will not seem natural to call the interval between $x$ and $y$ of really different size than that between $x$ and $z$. Now, it appears that an interval seems either to be one of difference or of identity. If it seems to be one of difference, it is indistinguishable from intervals of difference, and from those only, while all intervals of identity seem of the same magnitude. Hence, if our criterion of the genuine equality of two intervals is that all the intervals which seem identical with either seem identical with the other, if an interval be subliminal, it is genuinely identical in magnitude with the zero-interval. We thus obtain the result that the interval between $y$ and $z$, whose difference from zero we wish to secure, is genuinely identical in megnitude with a zero-interval. To avoid this, we limit our discussion at first to positive supraliminal intervals.

As we said above, we regard two intervals as genuinely equal " when and only when all the intervals which are indistinguishable from (i.e. seem neither greater nor less than) either are indistinguishable from the other. Genuine equality is, as mas readily be seen, a reflexive, symmetrical and transitive relation. It is possible, therefore, to group all positive supraliminal intervals into naturally exclusive sets, such that no member of any set is genuinely equal to a member of another set, and every member of a set is genuinely equal to every member of the set. The relation betiveen tivo terms which consists in their being separated by some interval belonging to one of these sets we shall call the vector assoeiated with the set. We are able, then, to regard any positive supraliminal interval as an instance of one of these vectors. We can next define the vector corresponding to a subliminal interval as follows : since all intervals representing a vector are equal, we should naturally regard the difference of an interval belonging to one vector from an interval belonging to another vector as independent with respect to its magnitude of the particular intervals chosen from these vectors. Now, the difference of two intervals may be readily defined when their upper ends coincide as the interval from the lower end of the interval from which the subtraction is to be made to the lower end of the subtracted interval. Furthermore, every subliminal interval, if its upper end does not lie in the neighbourhood of the maximum possible magnitude of sensations of the appropriate sort, may be regarded in the above manner as the difference of two supraliminal intervals with coincident upper ends. In the neighbourhood of the maximum possible magnitude of sensations of the appropriate sort, every subliminal interval may be regarded as the difference in an analogous

" Cf. "Studies in Synthetic Logic." 
sense of two intervals with coincident lower ends. It will be found, then, that the vector corresponding to a subliminal interval may be defined in terms of two supraliminal vectors whose difference it forms, as the relation between two terms when one either first ascends an interval belonging to one, and from that point descends an interval belonging to the other, or first descends an interval belonging to the second, and then ascends an interval belonging to the first. Now, we wish to confine our discussion to vectors made up of positive intervals. To this end, it is necessary that the subtracted vector should be smaller than that from which it is to be subtracted. We need next, therefore, a criterion of the relative magnitudes of trwo vectors.

In looking for such a criterion. we shall suppose it to be axiomatic that in any intrinsic comparison of differences between intervals, a subliminal or unnoticeable difference is always to be treated as less than a noticeable one. This being the case, if an interval be indistinguishable from some interval noticeably greater than another, it is necessarily greater than the other one-for, if the interval $R$ be indistinguishable from the interval $\mathrm{S}$, which is noticeably greater than $\mathrm{T}$, either $\mathrm{R}$ is subliminally greater than $\mathrm{S}$, or equal to it, or only subliminally less than it. In the first two cases, the naturalness of supposing $R$ greater than $T$ is obvious at once; in the latter case, the above principle will render it obvious. Another similar condition which determines that $R$ is greater than $\mathrm{T}$ is that there should be an interval $\mathrm{S}$, indistinguishable from $\mathrm{T}$, but noticeably less than $R$. It is logically demonstrable that one of these two criteria determines that an interval $R$ is greater than an interval $T$, or else that $T$ is greater than $R$ when, and only when, $R$ and $T$ are not genuinely equal, in accordance with our former definition. We may define $R$ as greater than $T$, then, when one of the two conditions just stated is satisfied.

We have now a complete definition of the class of intensity-vectors belonging to any given range of sensations. In the next section of this paper, we shall cover the same ground we have just been covering in a stricter manner, with the aid of the symbolism of the Principia Mathematica. If $\phi(x, y, u, v)$ stand for, "The difference between $x$ and $y$ in a given respect seems less than that between $u$ and $v$," then we shall use $I d_{\phi}$ to stand for the relation of genuine equality in the appropriate set between positive supraliminal intervals ; * $\dot{s}^{\prime \prime} \mathrm{D}$ ' $\overrightarrow{\mathrm{Id}}_{\phi}$ will be the class of

* We shall regard an interval as simply the ordered couple formed by its upper and lower extremity. 
positive supraliminal vectors: $\mathrm{De}_{\phi}$ stands for the relation, "less than," among positive supraliminal intervals, and $\mathrm{Vc}_{\phi}$ is the class of all intensityvectors. $\mathrm{Vs}_{s_{\phi}}$, which we shall later define in terms of $\mathrm{Vc}_{\phi}$ is a class of relations which will form part of $\mathrm{Vc}_{\phi}$ when $\phi$ has the particular kind of value just attributed to it, but which, unlike $\mathrm{Vc}_{\phi}$, will always consist of mutually exclusive vectors-i.e. of vectors such that no two distinct ones have a common beginning from which they reach to a common end-whatever properties $\phi$ may have.

We are now in a position to consider the problem of measurement itself. To this end, we define two vectors, $R$ and $S$, as having the ratio $\mu / \nu$ in a class of vectors $\kappa$ (which in the case of the measurement of sensoryintensities, will be the $\mathrm{Vs}_{\phi}$ just considered) if there is a vector $\mathrm{T}$ belonging to $\kappa$ such that if we start from a member of the field of $T$ and take successively $\mu$ steps belonging to $T$, we sometimes take one step belonging to $R$, while similarly, $v$ successive steps belonging to $T$ sometimes cover the the same ground as one step belonging to $\mathrm{S}$. This method of defining the ratio of two vectors in terms of a common submultiple, instead of in terms of a common multiple, as in the Principia, is chosen for the reason that we do not wish, for example, the existence of a loudness $\frac{9,999,999,999}{10,000,000,000}$ as great as that of the fulling of a pin to depend on that of a loudness $9,999,999,999$ times as great as that of the falling of a pin.

Each vector will bear various ratios to other vectors: it will be ten times this vector, twice that, half the other, and so on. We have seen, however, that in the case of sensation-intensities, no non-zero interval can be multiplied by an arbitrarily great numerical factor. The class of ratios, that is, which a given vector bears to other vectors, has, in general, a lower limit or minimum in the scale of real numbers which will be distinct from zero. In case there is a maximum vector to which the vector to be measured bears a ratio, this ratio will be the minimum of the ratios which the vector to be measured bears to other vectors, but, in general, a vector will not bear to any vector a ratio which is the minimum or lower limit of the ratios which it bears to other vectors, or, as we shall call it, its index. This index we shall represent by $\operatorname{Ind}_{x}{ }^{\prime} R$, where $R$ is the vector to be measured, and $\kappa$ the class of vectors in terms of which the measurement takes place. The index of a vector may roughly be taken to represent its measure in terms of the greatest possible vector of the set, but a vector can be measured by its index whether such a greatest possible vector exist or not. Since the series of real numbers is Dede. kindian-i.e. since, whenever we divide it into two clesses, so that every term of the one is greater than every term of the other, either the former 
class will have a minimum, or the second a maximum-every vector will have an index, and it is easy to show that no vector can have more than one index.

We have now found a way to measure such things as sensation-intervals; our next task is to discover a way to measure such things as sensations. Now, the natural measure of a sensation is the index of an interval stretching to it from a sensation of zero intensity. There may not be, however, a sensation of zero intensity. In such a case, the natural thing to do would seem to be to approximate to what the interval between this non-existent sense-datum of zero intensity and the given sensation would be, supposing we were wrong in judging data of zero intensity not to exist, by taking successively less and less intense sensations, measuring the intervals between these and the given sensation, and taking the upper limit or maximum of the values so obtained. Now, a sensation is less intense than a given sensation when the interval from it to the given sensation is positive, and the degree of their difference in intensity is measured by the index of their interval, so the natural measure of the intensity of a given sensation is the upper limit of the indices of intervals having it as their upper boundary. This we shall represent by Meas $_{\kappa}{ }^{\prime} x$, where $x$ is the sensation to be measured, and $\kappa$ is the class of vectors by which it is to be measured. It should be noted that in any system of measurement bounded at both ends, Meas ${ }_{k}$ will enable us to measure the position of any given term-it is not confined in its application to sensation-intensities. It should also be noted that the measure of any term in an unbounded system of measurement is zero, as every vector can be repeated an infinite number of times, and hence the lower limit of the ratios it bears to other vectors is zero. Therefore, since the measure of a term is the limit of a set of indices of vectors, it also must be zero. In any system each term will have one, and only one measure, but the same measure may belong to different terms. In such a case, if $\kappa$ is of the form $\mathrm{Vc}_{\phi}$ or $\mathrm{Vs}_{\phi}$, where $\phi$ is the relation between $x, y, u$, and $v$, when the intensity-interval between $x$ and $y$ seems algebraically less than that between $u$ and $v$, we shall say that two different terms having the same measure are of the same intensity, and that a sensation-intensity is the class of all terms having some given measure. The measure of an intensity is the measure of its terms. The relation between an intensity and its measure is one-one.

It will not be a necessary consequence of the definition of the measure of a vector that the measure of a vector containing an interval formed by taking steps belonging to two vectors $R$ and $S$, successively will be the sum of the measures of $R$ and $S$. We can easily obtain a new definition 
of the measure of a vector, however, which will always have this desired property. This reguires first the formation of a new class of vectors, which we shall term $R_{e} g_{\kappa}$, as a function of the class of vectors $\kappa$ with which we started. The measure of one of these, say $R$, will be designated Dist $_{x}$ 'R. We shall also define $\mu_{x}$ as the relation between a member of $\operatorname{Reg}_{\kappa}$ and an $\mu$-th submultiple of it ( $\mu$ being any real number). We shall prove that it follows from our definitions that any vector which is the $\mu$-th multiple of a $\nu$-th multiple of another vector is the $(\mu . \nu)$-th multiple of the second vector. The details of the definitions of these various notions are, however, of no special interest to the general reader, so I shall reserve them for the technically logistical portion of this paper.

Finally, we shall show how it is possible to remove the limitation of our system of measurement to systems of measurement with definite maxima or upper limits, and at the same time construct a method of measurement of sensation-qualities and intensities which is in certain cases more natural than that just given. Since all ranges of sensationqualities and intensities are bounded above, it is always possible to make the "maximum possible interval" our standard of measurement, and in the case of certain sensation-qualities, such as chroma, this seems the most natural standard to take--for example, we say that this patch of colour is of the highest possible degree of saturation, that one is only half saturated, and so on. In the case of most ranges of sensation-intensity, such as the scale of loudnesses, such a method of measurement seems highly artificial. It does not seem natural to measure the ticking of a watch in millionths of a boiler-factory-power. The interval which most psychologists have taken as a standard in such instances as these is the just-noticeable interval. We do not know, however, that all just-noticeable intervals are equal, nor yet that there are any just-noticeable intervals. We shall choose the first just-noticeable interval as our standard of measurement, and we shall avoid the assumption that there is some single definite just-noticeable interval, just as we avoided the assumption that there was any single, definite, greatest possible interval, by making the class of intervals which are less than just-noticeable-that is, are subliminal-our real standard of measurement. Now, all intervals which relate sense-data not noticeably more intense than any other sense data* are subliminal. We have now on our hands, therefore, the task of finding a way to measure terms of the fields of a class of vectors in terms of a cer-

- It may be necessary in some cases to exclude sensations of zero intensity from conside ration here and elsewhere for reasons analogous to those which previously led us to defer our consideration of subliminal intervals till after supraliminal intervals had been discussed. 
tain portion of these fields-namely, in the case of sense-intensities, in terms of the class of subliminal sense-data.

Now, we can use the system of measurement already developed to find the indices of all those portions of vectors which relate subliminal data to subliminal data, or, in general, data of a given portion of the fields of the class of vectors to data of the given portion of the fields of the class of vectors, in terms of the class of all such vectors. Let us regard the index of such a portion of the vector as a property of the whole vector, and not merely of the part for which it is primarily defined. Let us measure any vector $R$ of the original set by finding some $\mu$-th part of it to which an index has already been given by the method just indicated, and associating with $R$ a quantity $\mu$ times the value of this index. This quantity will, in general, depend on $\mu$. Let us call the relation of any such quantity to $R$, Inx $_{\kappa, a}$, where $\kappa$ is the class of vectors originally taken, and $a$ is the class of members of the fields of these vectors in terms of which all the members of the fields of these vectors are to be measured. Just as we previously defined the measure of a term to be the maximum or upper limit of the indices of intervals leading up to it, so we now define the measure of a term in terms of $\kappa$ and $\alpha$ as the maximum or upper limit of the values of the $\operatorname{Inx}_{x, a}$ 's of intervals leading up to it. This we shall call the Meas $x_{x, a}$ of the term in question. From this point on, the development of this theory of measurement runs precisely parallel to that of our previous theory.

At the end of this paper, we shall give a proof that under certain conditions which we there state, our method of measurement gives substan. tially the same results as that of the Principia. This portion of the paper is of a merely technical interest, in that it correlates this work with what has previously been done on the subject. It had better be omitted on a first reading of this paper.

1. We shall now cover the same ground we have already covered in the introduction in a strictly rigorous and logical manner, with the help of the symbolism of the Principia Mathematica. As we saw in the introduction, an experience which, as far as we are concerned, may be regarded as lying at the foundation of all our measurements of, for example, brightnesses, is, "The interval between $x$ and $y$, considered with reference to their brightness, seems algebraically less than that between $u$ and $v$," where all ascending supraliminal intervals-i.e. intervals between a sensedatum and another sense-datum of noticeably greater intensity, are regarded as positive. Let us call the above proposition $\phi(x, y, u, v)$. The thing that we should normally call the interval between $x$ and $y$ is the 
ordinal couple $x \downarrow y$. Now, we shall have occasion to regard $\phi$ as a dyadic relation between intervals rather than as a tetradic relation among brightness-sensations. On this account we shall make the following definition :

(1) $\quad \mathrm{Cp}_{\phi}=\hat{\mathrm{R}} \hat{\mathrm{S}}\{(\exists x, y, u, v) \cdot \mathrm{R}=x \downarrow y \cdot \mathrm{S}=u \downarrow v \cdot \phi(x, y, u, v)\} \quad \mathrm{Df}$

If $\phi$ is the relation mentioned above, $\mathrm{Cp}_{\phi}$ is the relation between a given brightness-interval and a brightness-interval which seems greater than it-algebraically greater, that is. We wish, however, to limit ourselves to the discussion of positive intervals. We obtain this result as follows: we first limit $\mathrm{Cp}_{\phi}$ to positive supraliminal intervals-that is, to intervals which bear the relation $\mathrm{Cnv}{ }^{\prime} \mathrm{Cp}_{\phi}$ to some interval of the form $x \downarrow x$. Then we form from this relation the one defined by means of the following definitions as $\mathrm{Id}_{\phi}$

$$
\begin{aligned}
& \mathrm{P}_{\mathrm{se}}=(-\mathrm{P}-\breve{\mathrm{P}})\left[\mathrm{C}^{\prime} \mathrm{P} \quad\right. \text { Df } \\
& \mathrm{P}_{\mathrm{s}}=\left(\overrightarrow{\overrightarrow{\mathrm{P}_{\mathrm{se}}}} \mid \overrightarrow{\mathrm{P}_{\mathrm{se}}}\right) \hat{\zeta} \mathrm{C} \cdot \mathrm{P} \quad \text { Df } \\
& \mathrm{Id}_{\phi}=\vdots \mathrm{Cp}_{\phi}\left[\hat{\mathrm{R}}\left[(\exists x) .(x \downarrow x) \mathrm{Cp}_{\phi} \mathrm{R}\right]\right\}_{\mathrm{s}} \quad \mathrm{Df}
\end{aligned}
$$

$I d_{\phi}$ is, then, the relation between two supraliminal positive brightnessintervals when they agree in every respect when they are compared with other brightness-intervals in respect to their magnitude. D ' $\overrightarrow{\mathrm{Id}}_{\phi}$ will, therefore, be the result of sorting out the class of all positive supraliminal brightness-intervals into classes each containing all the intervals of a given magnitude. $\dot{s}$ "D ' $\vec{I}_{\phi}$ will be the class of all positive supraliminal vectors-of all relations, that is, which connect pairs of sensations such that the brightness-interval between the members of such a pair is of a given fixed size for each member of $\dot{s}$ " $D^{\prime} \overrightarrow{I d}_{\phi}$ chosen, and is positive and supraliminal.

Now, one brightness-interval is less than another if it is either noticeably less than some brightness-interval indistinguishable from (i.e. neither noticeably greater than nor noticeably less than) the other, or is

\footnotetext{
* Cf. the discussion of indistinguishability and genuine identity in the introduction and Studies in Synthetic Logic.
} 
indistinguishable from something noticeably less than the other.* Let us define this relation, as it applies to supraliminal positive brightness. intervals, as follows:-

$$
\begin{array}{ll}
\mathrm{P}_{\mathrm{dc}}=\mathrm{P}\left|\mathrm{P}_{\mathrm{se}} \cup \mathrm{P}_{\mathrm{se}}\right| \mathrm{P} & \mathrm{Df} \\
\mathrm{Dc}_{\phi}=\left\{\mathrm{Cp}_{\phi}\left[\hat{\mathrm{R}}\left[(\exists x) \cdot(x \downarrow x) \mathrm{Cp}_{\phi} \mathrm{R}\right]\right\}_{\mathrm{dc}}\right. & \mathrm{Df}
\end{array}
$$

$\mathrm{Dc}_{\phi}$ is the desired relation. Now, one criterion of the equality of two subliminal or supraliminal positive intervals is, as we have seen, that they both can be formed by going up from some sensation by a step of a certain determinate size, and then down by a smaller step of another determinate size, or else by first taking a downward step of the smaller size, and by then ascending from the point just reached by a step of the larger size : that is, the relation between two terms connected by such an interval will be of the form $\breve{S}|R \cup R| \breve{S}$ where $R$ and $S$ are both mem-

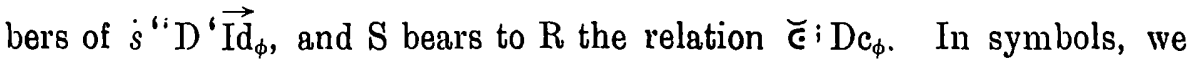
have the following definition:

$$
\mathrm{Vc}_{\phi}=\hat{\mathrm{T}}\left\{(\exists \mathrm{R}, \mathrm{S}), \mathrm{S}\left[\left(\widetilde{\epsilon} ; \mathrm{Dc_{ \phi }}\right)\left[\dot{s}^{\prime \prime} \mathrm{D} \cdot \overrightarrow{\mathrm{Id}}_{\phi}\right] \mathrm{R}, \mathrm{T}=\breve{\mathrm{S}}|\mathrm{R} \cup \mathrm{R}| \overline{\mathrm{S}}\right\}\right.
$$

$\mathrm{Ve}_{\phi}$ is, then, the class of brightness-vectors. We need to write

and not

$$
\begin{gathered}
\mathrm{T}=\overline{\mathrm{S}}|\mathrm{R} \cup \mathrm{R}| \overline{\mathrm{S}}, \\
\mathrm{T}=\overline{\mathrm{S}} \mid \mathrm{R} \text { nor } \mathrm{T}=\mathrm{R} \mid \overline{\mathrm{S}},
\end{gathered}
$$

* These two criteria are mutually irreducible. A sensation-interval of mayimal magnitude will not be indistinguishable from something noticeably greater than a subliminally smaller interval, while it will be noticeably greater than something indistinguishable from the latter interval. In the same way, a sensation-interval of minimal magnitude will not bo indistinguishable from any interval noticeably less than a subliminally greater interval, but will be noticeably less than something indistinguishable from the latter interval. The same sort of a statement may be made with reference to sensations, considered as to their intensity. The statement to the contrary in the Studies in Synthetic Logic already referred to is simply false, and arose from the author's not considering the fact that all ranges of sensory qualities or intensities have, roughly speaking, a maximum, If the int ' $R$ of that paper be defined as $\left\{\bar{\epsilon}_{i}\left(R\left|R_{s c} \cup R_{s e}\right| R\right)\right\}\left[\lambda_{R}\right.$ instead of as $\left\{\bar{\epsilon}_{j}\left(R_{s e} \mid R\right)\right\}\left[\lambda_{1 k}\right.$, the hypothesis

$$
R\left|R_{\mathrm{se}} \cup \mathbf{R}_{\mathrm{se}}\right| \mathrm{R}_{\mathrm{e}} \text { Trans }
$$

be substituted for

$$
R_{s e} \mid R \in T r a n s \text { or } R\left|R_{s e}\right| R \in R \text {, }
$$

and everything written about the hypothesis $R\left|R_{\mathrm{se}} \mathbf{C} R_{\mathrm{Be}}\right| R$ be struck out as unnecessary, since int ' $R$ will, as now defined, always be connected, the paper will be correct. The hypothesis $R\left|R_{\text {se }} \boldsymbol{C} \mathbf{R}_{\mathrm{se}}\right| \mathbf{R}$ will be satisfied everywhere except, so to speak, in the neighbourhood of the top of the scale of sensation-intensities, if $R$ be the relation, "noticcably more intense than." 
since, to put it crudely, we may be able to take a step of the size $a-b$ downwards or upwards when we are unable to take a step of the size $a$ in the given direction, where $a$ is less than half the length of the whole sensation-range, while we are always able to take a step of size $a$ either upwards or downwards from any given sense-datum. But even so, $\mathrm{V} c_{\phi}$ is not yet quite the class of sensation-intensity vectors we are looking for in order to apply our later theory of measurement, although it could, it is true, be used for that purpose as it stands. It will not be made up of mutually exclusive vectors in the case where $\phi$ is the relation which we have assumed it to be in the case of sensation-intensities. That is, it will be possible to go from one sensation to another, in general, by several distinct vectors. We would naturally suppose, however, that as the vectors which form the basis of our further theory of measurement represent, roughly, distances, that it would be impossible for two vectors to overlap. We get around this difficulty by defining in terms of $\mathrm{Vc}_{\phi}$ a class of vectors which would naturally be called intensity-vectors, or, in the case now under consideration, brightness-vectors, which will, by its very definition, be made up of mutually exclusive vectors. To this end, we first define the overlapping of two relations as the relation which holds between them when they both relate a given pair of terms together. In symbols, this becomes

$$
\text { Ov }=\hat{P} \hat{Q}\{\dot{\exists} ! P \cap Q\} \quad \text { Df }
$$

Then we fuse together any members of $\mathrm{Vc}_{\phi}$ which can be connected by a chain of overlappings, by the process indicated in the following definition:

$$
\mathrm{Vs}_{\phi}=\dot{s} " \mathrm{D} \cdot \overrightarrow{\left(\mathrm{Ov}\left[\mathrm{Vc}_{\phi}\right) *\right.} \mathrm{Df}
$$

$\mathrm{Vs}_{\phi}$ is called the class of separated vectors (i.e. in the present case, of separated brightness-vectors). It is useful on account of such conditions as the following: it is possible under all circumstances to go from a given sensation to one whose intensity is greater by $a$ units, ${ }^{*}$ by going up $a+b$ units in the scale of sensations, arranged as to their intensity, and then descending $b$ units, or else by going down $b$ units, and then ascending $a+b$ units, if $a+b$ is less than $\frac{1}{2} c$, where $c$ is the greatest number of units by which tro sensations can differ in intensity in the appropriate respect, while, if $a+b>\frac{1}{2} c$, this is only possible under certain condi-

* I speak as if we were able to measure sensations at this stage, although in fact we are not, since to express this point in strictly exact language would involve an intolerable and confusing prolixity. 
tions. Nevertheless, if it sometimes is possible, with a certain determination of $b$, to make a step $a$ units in length by going $b$ units down and $a+b$ units up, and it always is possible to make a step $a$ units in length by the same process when $b$ is given another determination, it would be most unnatural to regard these two processes as determining distinct vectors.

2. We now come to that part of our discussion which has most directly to do with measurement. We shall first define the ratio between two relations belonging to a given class (i.e. in the case of sensation-intensities, between tro intensity-vectors) in the sense which will be relevant in this paper, and derive a few allied classes and relations from it. I shall say that if $\mathrm{R}$ and $\mathrm{S}$ both belong to $\kappa$, they have the $\kappa$-ratio $(\mu / \nu)_{\kappa}$, if there is. a relation $\mathrm{P}$ which belongs to $\kappa$, such that

\section{$\exists ! \mathrm{P}^{\mu} \dot{\mathrm{n}} \mathrm{R}$ and $\dot{\exists} ! \mathrm{P}^{\nu} \dot{\cap} \mathrm{S}$}

In symbols, we have

$$
(\mu / \nu)_{\kappa}=\hat{R} \hat{S}\left\{(\exists P) . P, R, S \epsilon \kappa \cdot \exists ! P^{\mu} \dot{n} R . \exists ! P^{\nu} \dot{n} S\right\} \quad D f^{*}
$$

The definition of $\mu / \nu$ in the Principia will not do for us, because there, if $\mathrm{R}$ is to have to $\mathrm{S}$ the relation $\mu / \nu$, where $\mu / \nu$ is expressed in its lowest terms, we must have $\dot{\exists} ! R^{\nu} \dot{n} S^{\mu}$, so that it would be impossible for us to say, for example, that a fog-horn sounds two-thirds as loud as a boilerfactory, without assuming that there is something sounding twice as loud as a boiler-factory. On the other hand, it will be seen that, on our definition, it may very well be that a fog-horn makes a noise two-thirds as loud as a boiler-factory, without there being any noise twice as loud as that of a boiler-factory.

An important notion connected with $(\mu / \nu)_{\kappa}$ is that of the class of $\kappa$-ratios which a given relation $R$ bears to other relations. I shall define $R t_{k}$ as follows:

$$
R t_{\kappa}=\hat{S} \hat{R}\left\{(\exists \mathrm{M}, \mu, \nu) . \mathrm{S}=(\mu / \nu)_{\kappa} \cdot \mu, \nu \neq 0 . \mathrm{R}(\mu / \nu)_{\kappa} \mathrm{M}\right\} \quad \mathrm{Df}
$$

Then $\overrightarrow{R t}_{x}$ ' $R$ is this class. In a vector family, $\dagger$ the analogue of $\overrightarrow{R t}_{\kappa}$ ' $R$ -i.e. in a submultipliable connected vector-family, $\overrightarrow{R t}_{\kappa}$ ' $R$ itself-contains ratios less than any given ratio. In a class of relations which serve as

* This is not the $(\mu / v)_{k}$ used in the discussion of cyclical systems in the Principia.

† Cf. Principia Mathematica, Part VI, Section B.

SER. 2. VOL. 19. No. 1363. 
the basis of a system of measurement with a maximum, the ratios, taken in the sense of the Principia, which are the analogues of $\overrightarrow{R t}_{\kappa}$ ' $R$, determine as their "lower limit" in the series of real numbers a number which, in general, will not be zero, which we shall call the $\kappa$-index of $\mathrm{R}$, or $\operatorname{Ind}_{\kappa}{ }^{\prime} R$. To define this, we need first to determine the analogue of one of our ratios in the system of the Principia. If $(\mu / \nu)_{\kappa}$ be one of our ratios, its analogue will be $\mathrm{Eq}_{k}{ }^{\prime}(\mu / \nu)_{k}$, where $\mathrm{E}_{\mathrm{q}_{k}}$ is defined by

$$
\mathrm{E} \mathrm{q}_{\kappa}=\hat{\mathrm{R}} \hat{\mathrm{S}}\left\{(\exists \mu, \nu) \cdot \mathrm{R}=\mu / \nu . \mathrm{S}=(\mu / \nu)_{\kappa}\right\} \quad \mathrm{Df}
$$

Then $\operatorname{Ind}_{\kappa}$ will be defined by

$$
\operatorname{Ind}_{\kappa}=p\left|\left(\overrightarrow{\mathrm{H}}_{\epsilon}\right)\right|\left(\mathrm{Eq}_{\kappa}\right)_{\epsilon} \mid \overrightarrow{\mathrm{Rt}}_{\kappa} \quad \text { Df }
$$

This will always make $\operatorname{Ind}_{\kappa}$ ' $\mathrm{R}$ a real number or' $\Lambda$, by the definition of a real number given in $* 310^{*}$ of the Principia. It follows from $* 72-12-15-16, * 71-25$ that $\operatorname{Ind}_{\kappa}$ is a one-many relation: that is, no relation has more than one $\kappa$-index. We are enabled to compare incommensurable members of $\kappa$ by their $\kappa$-indices, for their $\kappa$-indices express, roughly speaking, their magnitude in terms of that of the maximum of all the $\kappa$-vectors. It should be noted, however, that we have nowhere assumed that $\kappa$ contains any greatest $\kappa$-vector: indeed, we have assumed nothing at all about $\kappa$ except that it is a class of relations.

$\operatorname{Ind}_{\kappa}$ enables us to assign a real number to every member of $\kappa$-that is, for example, to every vector between sensations considered with regard to their intensities or qualities. We do not merely wish, however, to measure vectors between sensations considered with regard to their intensities and qualities, but also to measure these intensities and qualities themselves. The measure of the intensity of a sensation is, roughly speaking, the index of its difference from a sensation of zero intensitythat is, it is the upper limit or maximum (taken in the series of real numbers, in their natural order) of the indices of the vectors leading up to it from sense-data of smaller intensity. If $\kappa$ stands for the class of separated intensity-vectors, and $\mathrm{Meas}_{\kappa}$ is to stand for the relation between the measure of the intensity of a sense-datum and a sense-datum possessing this measure, we get, in symbols

$$
\operatorname{Meas}_{\kappa}=\left\{\operatorname{limax}_{\Theta^{\prime}}\left|\left(\operatorname{Ind}_{\kappa}\right)_{e}\right|(\widetilde{\sigma})_{\epsilon} \overleftarrow{\varepsilon}\right\}\left\lceil s^{\prime} \mathrm{C}^{\prime \prime} \kappa \quad\right. \text { Df }
$$

* References beginning with a $\%$ are to paragraphs and theorems in the Principia Mathematica. 
By $* 310-1, * 207-41, * 204-1, * 72-12-15, * 71-25-26$ of the Principia, Meas $_{\kappa}$ is a one-many relation. It obviously correlates with the whole of $s^{\prime} C^{\prime \prime} k$ a part of $C^{\prime} \theta^{\prime}$, or the class of all positive real numbers and zero. Meas $s_{\kappa}$ will not, in general, be one-one.*

From Meas $\mathrm{s}_{\mathrm{k}}$ we may derive a class of vectors or relations connecting members of $s{ }^{\prime} \mathrm{C}$ " $\kappa$ which will al ways have certain very important properties, quite independent of those of $\kappa$. I shall define this class, which I shall call $\mathrm{Reg}_{\kappa}$, as follows

$$
\begin{aligned}
\text { Dist }_{\kappa} & =\hat{\mathrm{R}} \hat{\mu} ; \mathrm{R}=\mathrm{Meas}_{\kappa} ; \mu+{ }_{\prime \prime} \quad \text { Df } \\
\operatorname{Reg}_{\kappa} & =\mathrm{D}{ }^{\prime} \text { Dist }_{\kappa}-\imath \cdot \dot{\mathrm{A}}
\end{aligned}
$$

The measure of a member of $\operatorname{Reg}_{x}$ is the value of $\mu$ for which this member

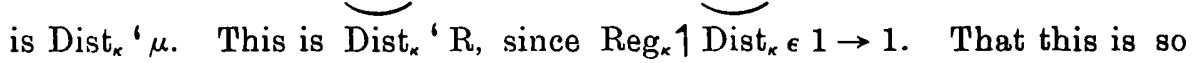
may be proved as follows : it results from the form of the definition of Dist $_{\kappa}$ that Dist $_{\kappa} \in 1 \rightarrow$ Cls, and hence that $\operatorname{Reg}_{\kappa} 1$ Dist $_{\kappa} \in 1 \rightarrow$ Cls. Now let us suppose that Dist $_{\kappa}{ }^{\prime} \mu=$ Dist $_{\kappa}{ }^{\prime} \nu \neq i$ : i.e. that Dist $_{\kappa}{ }^{\prime} \mu$ and Dist ${ }^{\prime}$ ' are identical, and belong to $\operatorname{Reg}_{x}$. This gives, by definition,

$$
\overline{\operatorname{Meas}}_{\kappa} ; \mu+_{a}=\overline{\operatorname{Meas}}_{\kappa} ; \nu+_{a} \neq i
$$

From this we deduce

$$
\begin{aligned}
& (\exists \mathrm{R}, \mathrm{S}):(\exists \varpi, \rho) \cdot \mathrm{R} \overline{\operatorname{Meas}}_{\kappa} \varpi \cdot \varpi\left(\mu+_{a}\right) \rho \cdot \rho \mathrm{Meas}_{\kappa} \mathrm{S} \text { : } \\
& (\exists \sigma, \tau) \cdot \mathrm{R} \operatorname{Meas}_{\kappa} \sigma \cdot \sigma(\nu+a) \tau \cdot \tau \text { Meas }_{\kappa} \mathrm{S},
\end{aligned}
$$

whence we obtain

$$
\begin{gathered}
(\exists \mathrm{R}, \mathrm{S}, \varpi, \rho, \sigma, \tau) \cdot \varpi \mathrm{Meas}_{\kappa} \mathrm{R} \cdot \sigma \operatorname{Meas}_{\kappa} \text { R. } \rho \mathrm{Meas}_{\kappa} \text { S. } \tau \text { Meas }_{\kappa} \mathrm{S} . \\
\varpi=\mu+a \rho \cdot \sigma=\nu+_{a} \tau .
\end{gathered}
$$

Since $\mathrm{Meas}_{\kappa} \in 1 \rightarrow \mathrm{Cls}$, and since $\mathrm{D}^{\prime} \mathrm{Meas}_{\kappa} \subset \mathrm{C}^{\prime} \theta^{\prime}$, by definition, it follows from $* 310-123, * 312-55-41$ of the Principia that $\mu=\nu$. Hence

* A condition which will make Meask one-one is

$$
\operatorname{Ind}_{\kappa}\left\lceil\kappa \in 1 \rightarrow 1::(\exists x): \cdot y \in S^{\prime} \mathrm{C}^{\prime \prime} \kappa \cdot \supset_{\nu}:(\exists \mathrm{R}): x \mathrm{R} y: z \mathrm{~S} y \cdot \mathrm{S} \in \kappa \cdot \supset_{z, \mathrm{~s}} \cdot \mathrm{S}\left(\operatorname{Ind}_{\kappa}{ }^{i} \Theta^{\prime}\right) \mathrm{R}\right.
$$

Ind $d_{\kappa}\lceil\kappa \in 1 \rightarrow 1$ is true if, for example, the members of $\kappa$, arranged in the natural order of their indices, form a series, as we should naturally expect when they are e.g. intervals between sensation-intensities defined as in the "Studies in Synthetic Logic" (not between sensations). The second part of this condition amounts to the assumption that there is a member of $s^{\prime} \mathrm{C}$ " $\kappa$ such that if $y$ is another member of $s^{\prime} \mathrm{C}^{\prime \prime} \kappa$, there is a member of $\kappa$ which relates this term to $y$, whose $\kappa$-index is less than that of $y$. 
$\operatorname{Reg}_{\kappa} 1$ Dist $_{\kappa} \in 1-1$. Since $\operatorname{Reg}_{\kappa}$ is the domain of this relation we can always speak of $\overline{D i s t}_{\kappa}$ ' $R$ if $R \in \operatorname{Reg}_{\kappa}$.

It is easy to prove that if $R, S$ and $R \mid S$ are all members of $R e g_{k}$, the measure of $R \mid S$ is the sum of the measures of $R$ and of $S$, or, in symbols,

$$
\vdash: R, S, R \mid S \in \operatorname{Reg}_{k} \cdot \partial_{R, S, k} \cdot \overline{\text { Dist }}_{\kappa} \cdot(R \mid S)={\overline{\text { Dist }_{k}}}^{\prime} R+{ }_{a} \overline{\text { Dist }}_{\kappa} \text { 'S }
$$

As a consequence of this, since $\operatorname{Reg}_{\kappa} 1$ Dist $_{\kappa} \in 1 \rightarrow 1$ we get

$$
\vdash: R, S, R|S, S| R \in R_{e g} \text {. ว. R|S = S |R }
$$

Relative multiplication, then, applied to members of $\operatorname{Reg}_{\kappa}$, is not only an associative operation, but a commutative operation, and may be regarded, roughly, as a kind of addition. This shows that the old opinion that only extensive magnitudes are subject to a commutative, associative operation of addition is erroneous. We can not only get a commutative, associative operation of addition which will apply to vectors such as the members of $\operatorname{Reg}_{\kappa}$, but also one which will apply to sensation-intensities, as we may now call the members of $D$ ' $\overleftarrow{M e a s}_{\kappa}$, where $\kappa$ is a class of separated intensityvectors, since they are classes of all the sense-data whose intensity has a given measure. This operation is simply the operation on $\alpha$ and $\beta$ which gives the $\gamma$ such that the measure of the members of $\gamma$ is the sum of the measure of the members of $\alpha$ and the measure of the members of $\beta$.

Another consequence of (17) is that if we define $\mu_{\kappa}$ as the relation which holds between $R$ and $S$ when they both belong to $\operatorname{Reg}_{x}$ and

一that is, if we put

$$
\overline{\text { Dist }}_{\kappa} \text { 'R }=\mu \times{ }_{a} \overline{\text { Dist }}_{\kappa} \text { 'S }
$$

$$
\mu_{\kappa}=\left\{\text { Dist }_{\kappa} ; \mu \times_{a}\right\}\left[\operatorname{Reg}_{\kappa} \quad \mathrm{Df}\right.
$$

we shall have

$$
\vdash:\left(\mu_{x}^{\prime} R\right) \mid\left(\nu_{x}^{\prime} R\right)=\varpi_{x} \cdot R \cdot \supset \cdot \mu+a^{\nu}=\varpi
$$

or, more generally,

$$
\vdash: \exists !\left[\left(\mu_{\kappa}{ }^{\prime} \mathrm{R}\right) \mid\left(\nu_{x}{ }^{\prime} \mathrm{R}\right)\right] \cap \varpi_{\kappa}{ }^{\prime} \mathrm{R} \cdot \partial \cdot \mu+{ }_{a} \nu=\varpi
$$

Moreover, since $\operatorname{Reg}_{\kappa} 1 \operatorname{Dist}_{\kappa} \varepsilon 1 \rightarrow 1$, and $\left(\mu \times_{a}\right) \mid\left(\nu X_{k}\right)=\left(\mu \times_{a} \nu\right) X_{a}$, we get

$$
\vdash: \exists \mu_{\kappa} \mid \nu_{x} \dot{i} \varpi_{k} \cdot \partial \cdot \mu \times_{a}{ }^{\nu}=\varpi
$$

These fropositions are the rough analogues of $* 356-33-54$ in the P'rincipiz. 
Let us next bring the discussion of measurement that we have just finished into correlation with that part of our theory that derived $\mathrm{Vs}_{\phi}$ from $\phi$. If $\phi$ be such a relation as, "The interval of saturation in color between $x$ and $y$ seems less than that between $u$ and $v$," the natural unit of measurement, as we said in the introduction, would seem to be a complete saturation, and the method of measurement which we have just elaborated would seem fairly natural and appropriate. The measure of the saturation of a given sensation $x$, will then be $\operatorname{Measv}_{s_{q}}{ }^{\prime} x$. It will be natural to say that two sensations have the same degree of chroma if they

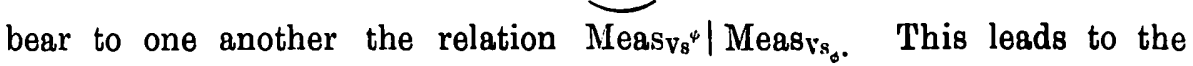
following definitions

$$
\begin{aligned}
& \text { Qual }_{\phi}=\mathrm{D} \cdot \overleftarrow{M e a s}_{\mathrm{r}_{\phi}} \text { Df } \\
& \mathrm{Mag}_{\phi}=\text { Measvs }_{s_{\phi}} \mid \epsilon \uparrow \text { Qual }_{\phi} \text { Df }
\end{aligned}
$$

Qual $\phi$ is the class of all degrees of saturation, in the particular case just discussed. It consists, however, of degrees of saturation considered as quantities, and though it may de facto coincide with the $\lambda_{R}$ of the Studies in Synthetic Logic, if $\mathrm{R}$ be taken to be the relation, "noticeably more saturated than," or with $\mathrm{D}^{\prime} \mathrm{sg}^{\prime}\left(\dot{s}^{\prime} \mathrm{V} \mathrm{s}_{\phi}\right)_{\mathrm{se}}$, it cannot, without the aid of complicated logical hypotheses, be proved identical with either.

$\mathrm{Mag}_{\phi}$ is the relation of the magnitude of a degree of saturation to the degree of saturation, and is one-one. If we so desire, we can define Dist $_{\phi}$ as $\hat{\mathrm{R}} \hat{\mu}\left\{\mathrm{R}=\overline{\mathrm{Mag}}_{\phi} ; \mu+_{a}\right\} \operatorname{Reg}_{\phi}$ as $\mathrm{D}^{\prime} \mathrm{Dist}_{\phi}-\iota ' \dot{\Lambda}$, and $\mu_{\phi}$ as

and the propositions

$$
\left\{\text { Dist }_{\phi} ; \mu X_{\mu}\right\}\left\lceil\operatorname{Reg}_{\phi}\right.
$$

$$
\vdash: \dot{\exists} !\left[\left(\mu_{\phi}{ }^{\prime} R\right) \mid\left(\nu_{\phi} \text { 'R }\right)\right] \dot{n} \varpi_{\phi} \text { 'R.J. } \mu+_{\alpha^{\nu}}=\varpi,
$$

and

$$
\vdash: \dot{\exists} ! \mu_{\phi} \mid \nu_{\phi} \dot{n} \varpi_{\phi} \cdot \supset \cdot \mu \times_{1,} \nu=\varpi \text {, }
$$

will be universally valid. $\operatorname{Reg}_{\phi}$ will consist of all intervals, positive, zero, and negative, between degrees of saturation, and if $R$ be any such interval, its measure will be $\overline{\text { Dist }}_{\phi}$ 'R. $\mu_{\phi}$ will be the proportion between a member of $\operatorname{Reg}_{\phi}$ and another, one $\mu$-th of its size.

3. In our previous work we have, roughly speaking, made the maximum of the magnitudes of all members of $\kappa$ our real unit of measurement : let us see how this measurement may be adapted to measurement by smaller units. The way to do this is to select a certain region of our 
scale of measurement which has a definite maximum, to measure the intervals lying in this region by the method given above, and to measure all intervals in general by finding fractions of them lying in this interval. To this end, we make the following definitions

$$
\begin{aligned}
& \mu \div \lambda=\mu \times{ }_{a} \mathrm{H}^{\prime}(1 / \lambda) \quad D \mathrm{f}
\end{aligned}
$$

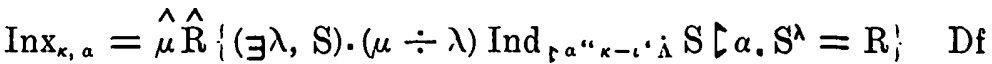

Here we use as our standard of measurement the greatest distance between members of $\alpha$, measured by members of $\kappa$, and not the greatest distance between members of $s^{\prime} C^{\prime \prime} \kappa$. Inx $x_{x, a}$ is not, in general, a onemany relation: its converse domain is $s^{\prime} \operatorname{Pot}^{\prime}\{\kappa \cap \hat{R}\{\exists ! R[a\}\}$ and its domain is included in the class of real numbers. We next define Meas $x_{x}$ a in terms of $\operatorname{Inx}_{\kappa, a}$, as we defined Meas $x_{\kappa}$ in terms of $\operatorname{Ind}_{\kappa}$, in the following manner

$$
\begin{aligned}
& \operatorname{Meas}_{\kappa, a}=\left\{\operatorname{limax}_{\Theta^{\prime}}\left|\left(\operatorname{Inx}_{\kappa, a}\right)_{e}\right|(\widetilde{O})_{e} \mid \overleftarrow{\varepsilon}\right\} \uparrow \\
& s^{6} \mathrm{C} \text { "Pot" }\{\kappa \cap \hat{R}\{\dot{\exists} ! \mathrm{R}[\alpha\}\} \quad \mathrm{Df}
\end{aligned}
$$

Notwithstanding the fact that $\operatorname{Inx}_{\kappa, a}$ is not, in general, one-many, Meas $_{\kappa_{1}, a}$ is always necessarily one-many.

We are now in a position to define Dist $_{\kappa, a}, \operatorname{Reg}_{\kappa, a}$ and $\mu_{\kappa, a}$ just as we defined Dist $t_{\kappa}, \operatorname{Reg}_{\kappa}$, and $\mu_{\kappa}$ : that is, as follows

$$
\begin{array}{rlrl}
\operatorname{Dist}_{\kappa, a} & =\hat{\mathrm{R}} \hat{\mu}\left\{\mathrm{R}=\overline{\text { Meas }}_{\kappa, a} ; \mu+_{a}\right\} & \mathrm{Df} \\
\operatorname{Reg}_{\kappa, a} & =\mathrm{D}^{\prime} \operatorname{Dist}_{\kappa, a}-\iota^{\prime} \dot{\mathrm{A}} & & \mathrm{Df} \\
\mu_{\kappa, a} & =\left\{\text { Dist }_{\kappa, a} ; \mu x_{a}\right\} \operatorname{Reg}_{\kappa, a} & & \text { Df }
\end{array}
$$

All the theorems which we proved concerning Meas $_{\kappa}$, Dist $_{\kappa}$, Reg ${ }_{\kappa}$, and $\mu_{\kappa}$ will remain valid if we alter $\kappa$ every time that it occurs as a subscript to $\kappa, a$.

Now, if $\phi(x, y, u, v)$ stands for " the brightness-interval between $x$ and $y$ seems less than that between $u$ and $v$," the theory of sensation-measurement developed in $\S 2$, though it is still applicable, does not seem appropriate nor natural : we do not usually take the greatest possible brightness, say, that of the sun, as our standard of brightness. Our usual way of measuring brightness seems to be, perhaps, to count the number of "thresholds" which lie between it and utter darkness. In all ordinary psychological work, this method of measurement is adequate, but it will 
not enable us to subdivide the step from one limen to the next into equal parts. Nevertheless, the limen is a very natural standard to use in the measurement of brightnesses. Since we do not wish to assume that all steps from a given sense-datum to a just brighter one are equal, we shall make the first interliminal difference our standard of measurement. We do this by taking $\mathrm{Vs}_{\phi}$ as the $\kappa$ and the class of sense-data lying in the first interliminal space as the $a$ of the theory of measurement which we have just developed. Now, a sense-datum lies in the first interliminal space with respect to brightness, or is of subliminal brightness, if no interval leading to it from below is supraliminal : that is, if it belongs to the field of $\dot{s}^{\prime} \hat{\mathrm{R}}\left\{(\exists x) .(x \downarrow x) \mathrm{Cp}_{\phi} \mathrm{R}_{\}}\right.$, but not to its converse domain. Let us define this class, and certain functions of it, as follows

$$
\begin{aligned}
\mathrm{Sbl}_{\phi} & =\overrightarrow{\mathrm{B}}^{\prime} \dot{\mathrm{s}}^{\prime} \hat{\mathrm{R}}\left\{(\exists x) \cdot(x \downarrow x) \mathrm{Cp}_{\phi} \mathrm{R}\right\} \quad \text { Df } \\
\mathrm{Quant}_{\phi} & =\mathrm{D}^{\prime} \mathrm{Gs}^{\prime} \mathrm{MeaSv}_{\mathrm{v}_{\phi}, \mathrm{Sb}_{\phi}} \text { Df } \\
\mathrm{Mgn}_{\phi} & =\mathrm{Meass}_{\mathrm{v}_{\phi}, \mathrm{Sbl}_{\phi}} \mid \epsilon \uparrow \operatorname{Quant}_{\phi} \quad \text { Df }
\end{aligned}
$$

$\mathrm{Sbl}_{\phi}$ is, in the case just considered, the class of all sensations of subliminal brightness. Qnant ${ }_{\phi}$ is the class of all degrees of brightness. $\mathrm{Mgn}_{\phi}{ }^{\prime} \xi$ is the quantitative measure of $\xi$ in terms of the brightnesslimen, if $\xi$ is a brightness. The further development of this theory of measurement runs precisely parallel to that of measurement in terms of the maximum interval.

4. The question which we have next to settle is that of the relation of our theory of measurement to that of the Principia. It is obvious that since the theory of the Principia applies only to kinds of measurement without definite maxima, while our theory of measurement, in the form it takes in $\$ 2$, only applies to kinds of measurement with definite maxima, the form of our theory with which we must compare that of the Principia is that developed in $\S 3$, which need not only apply to kinds of measurement with definite maxima. To carry out this comparison, we need to set some standard problem, and compare the answers which the two systems give to it. Now, the theory in the Principic starts from a certain sort of class of relations or vectors $\kappa$, and culminates in the definition of the real proportion $X$ among members of $\kappa$ as $X_{\kappa}$. Our definition of the real proportion $\mu$, among members of $\operatorname{Reg}_{\kappa, a}$ is $\mu_{x, a}$, and therefore a natural definition of the real proportion $\mu$, among members of $\kappa$ will be $\mu_{x, a}[\kappa$. The fact that the real proportion in the Principia appears in 
the form of a function of a relation, while ours appears as a function of a class, is due to the fact that the real numbers which are applied there are the $\dot{s}$ 's of the real numbers we apply. The natural way to put the question, "When does the theory of measurement developed in the Principia give the same results as that developed in $\$ 3$ of the paper?" is "When is the formula $\mu_{x, a}\left\lceil\kappa=\left(\dot{s}^{\prime} \mu\right)_{\kappa}\right.$ valid?"

Now, if $\alpha$ is a connected family, containing all the powers of its members, it follows from $* 381-24-42$ that

$$
(\mu / \nu)_{\kappa}=\hat{\mathrm{R}} \hat{\mathrm{S}}\left\{(\exists \mathrm{P}) \cdot \mathrm{P} \epsilon \kappa \cdot \mathrm{R}=\mathrm{P}^{\mu} \cdot \mathrm{S}=\mathrm{P}^{\nu}\right\},
$$

or, in extenso,

$$
\begin{aligned}
& 1: \text { : } \kappa \mathrm{FM} \text { conx. s'Pot" } \kappa(\kappa \cdot) \cdot \\
& (\mu / \nu)_{\kappa}=\hat{\mathrm{R}} \hat{\mathrm{S}}\left\{(\exists \mathrm{P}) . \mathrm{P} \epsilon \kappa \cdot \mathrm{R}=\mathrm{P}^{\mu} \cdot \mathrm{S}=\mathrm{P}^{\nu}\right\} .
\end{aligned}
$$

Since, if $\mathrm{R}=\mathrm{P}^{\mu}$ and $\mathrm{S}=\mathrm{P}^{\nu}, \mathrm{R}^{\nu}=\mathrm{P}^{\mu \times, \nu}=\mathrm{S}^{\mu}$, and since, under the above conditions, $\mathrm{R}, \mathrm{S}$, and $\mathrm{R}^{\nu}$, being powers of a member of $\kappa$, are members of $\kappa$, and hence not $\dot{\Lambda}$, we derive from $* 302-02$ and $* 303-01$ the conclusion,

$$
\vdash: \operatorname{Hp}(34) . \supset \cdot(\mu / \nu)_{\kappa} \Subset(\mu / \nu)[\kappa
$$

From (34), (35), and $* 333-42, * 351-1$, we get

$$
\vdash: \mathrm{Hp}(34) \cdot \kappa \in \mathrm{FM} \text { ap submult. } \cdot \cdot(\mu / \nu)_{\kappa}=(\mu / \nu)[\kappa
$$

Furthermore, it results from (34) und $* 336-41$ that

$$
\vdash: \operatorname{Hp}(34) \cdot R_{\epsilon} \kappa_{\partial} \cdot \supset \cdot(\mu / \nu)_{U_{\kappa} \leftarrow^{\circ}}=(\mu / \nu)_{\kappa}\left[U_{\kappa}{ }^{\prime} R\right.
$$

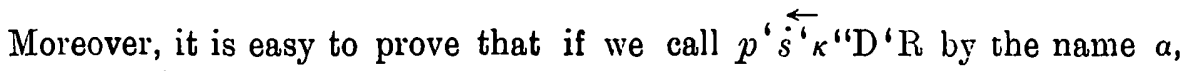
and if $P[\alpha$ and $Q[\alpha$ exist, then to say that $P$ bears the relation $(\mu / \nu)_{\kappa}\left[\overleftarrow{\mathrm{U}_{\kappa}} \mathrm{R}\right.$ to $\mathrm{Q}$ is equivalent to saying that $\mathrm{P}[a$ bears the relation $(\mu / \nu)_{L_{a}}$ " $\overleftarrow{U}_{a} \cdot R$ to $Q[a$, under the hypotheses that $\kappa$ is a connected family containing all the powers of its members, and that $R$ belongs to $\kappa_{\partial}$. In symbols we have

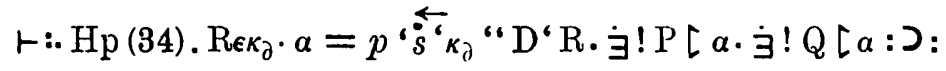

$$
\begin{aligned}
& P\left\{(\mu / \nu)_{\kappa}\left[\overleftarrow{U_{\kappa}} \cdot R\right\} Q . \equiv \cdot\left(P[\alpha)(\mu / \nu)_{L \alpha} \cdot \overleftarrow{U}_{\star} \cdot R(Q[\alpha) .\right.\right.
\end{aligned}
$$


Putting (38) and (36) together, we get

$$
\begin{aligned}
& \text { ト : } \operatorname{Hp}(38) \cdot H p(36): \supset: P\left\{(\mu / \nu)\left[\overleftarrow{U}_{\kappa}{ }^{\prime} R\right\} Q\right. \text {. } \\
& \equiv \cdot\left(P \left[(\alpha)(u / v) \tilde{L}_{\alpha} \| \overleftarrow{U}_{\alpha} R(Q[\alpha) .\right.\right.
\end{aligned}
$$

With the end of (39), (11), and (12), we obtain

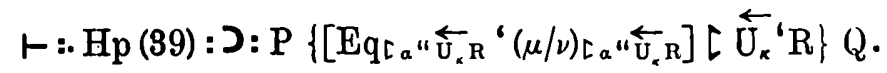

$$
\begin{aligned}
& \equiv \cdot \mathrm{P}\left\{\left[\alpha^{\dagger}(\mu / \nu)_{\left[\alpha^{\prime \prime} \overleftarrow{U}_{x} \cdot R\right.}\right\} Q\right.
\end{aligned}
$$

whence we deduce

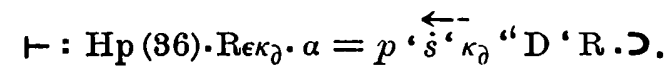

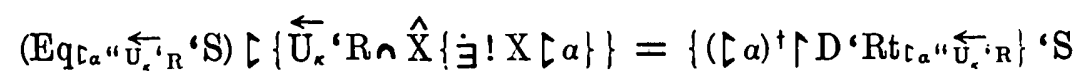

The question now arises, when will it be true that if $\mathrm{RU}_{\kappa} \mathrm{T}$,

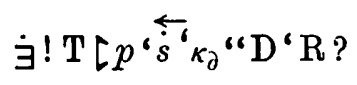

Now, if $\kappa$ is an initial family, it is not difficult to deduce from $* 335-15$ and the definition of a vector-family that $p$ ' $\overleftarrow{s} \kappa_{\partial}$ " $D$ ' $R$ is made up of all those things which bear such a relation as $\breve{\mathrm{P}}$ to everything bearing such a relation as $R \mid Q$ to init ' $\kappa$, where $P \epsilon \kappa_{\partial}$ and $Q \epsilon \kappa$. Now, by $* 335-17$, the relative product of two members of an initial family is a member of the same family (see also $* 331-23$ ), and hence, since

$$
x \breve{\mathrm{P}} \mid \mathrm{R} \text { init ' } \kappa \text {. ว. } x \breve{\mathrm{P}}|\breve{\mathrm{Q}}| \mathrm{R} \mid \mathrm{Q} \text { init ' } \kappa \text {, }
$$

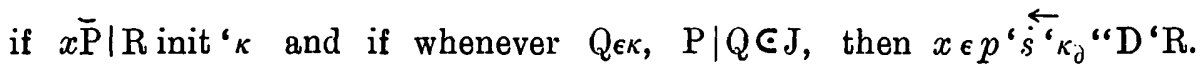

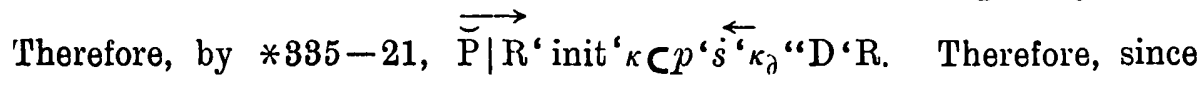

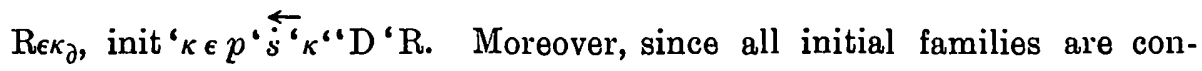
nected by $* 336-41$, if $\mathrm{RU}_{\kappa} \mathrm{T}$ there is a member of $\kappa_{\partial}$, say $\mathrm{S}$, such that $\mathrm{R}=\mathrm{S} \mid \mathrm{T}$. Since $\kappa \mathrm{C} 1 \rightarrow 1$ and all the members of $\kappa$ have a common converse domain, $T=\breve{S} \mid R$ and hence $T$ 'init' $\kappa$ is the same as $\breve{S}$ ' $R$ 'init $\kappa$,

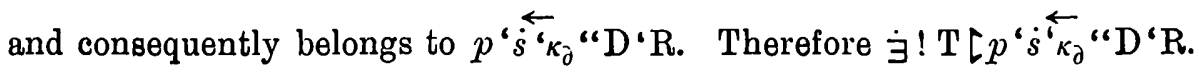
This gives us, by (41), $* 335-17$, and the definition of FM init,

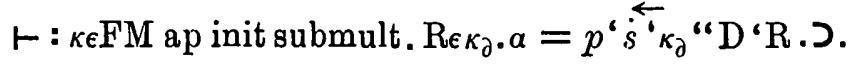

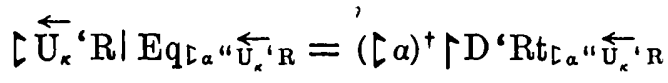


If $\dot{s}^{\prime} \kappa_{\partial}$ is a series, then no term can at once belong to $\mathrm{D}$ ' $\mathrm{R}$ and to $p ` \overleftarrow{s} \kappa_{\partial}$ "D $D$ 'R, by $* 202-503$. It may be deduced from $* 336-41$, moreover, that if $\mathrm{P}_{\epsilon} \overleftarrow{\mathrm{U}}_{x}{ }^{\prime} \mathrm{R}, \mathrm{D}^{\prime} \mathrm{P} C \mathrm{D}^{\prime} \mathrm{R}$. From this and what we have said above, we may conclude that $\left[a\right.$ " $\overleftarrow{U}_{\kappa} ' R=[a$ " $k-\imath \cdot \dot{\Lambda}$. From this, (42), and $* 334-32$, we get

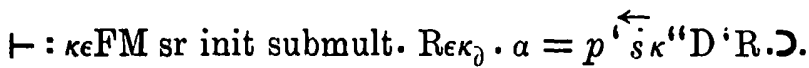

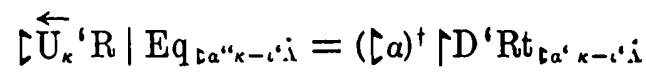

Now, it follows from this and (13) that $\operatorname{Ind}_{\Gamma_{a}}{ }_{k-1} \cdot \dot{ } \cdot \mathrm{\prime} S$ is the class of all those ratios which are less than any ratio which the member of $\overleftarrow{U}_{k}^{\prime}$ ' $R$ of which $S$ forms a part bears to any member of $\overleftarrow{U}_{\kappa}$ ' $R$ : by $* 352-72$, then, if $\alpha$ be a serial family, as we have supposed in (43), the member of $\overleftarrow{U}_{\kappa}^{\prime} R$ containing $S$ bears to $R$ (or to any member of $\overleftarrow{U}_{x}$ ' $R$ ) no ratio which is a member of Ind $d_{L_{a}}{ }_{x-1} \cdot \dot{i}$ 'S , and no member of $\kappa$ preceding the one containing $S$ bears $R$ such a ratio, while, by the definition of $\operatorname{Ind}_{L_{a}}{ }_{k-1} \cdot . \cdot$ and $* 352-72$, any member of $\kappa$ following the one containing $S$ does bear $R$ such a ratio, if any. Hence it is easy to prove that if the rational

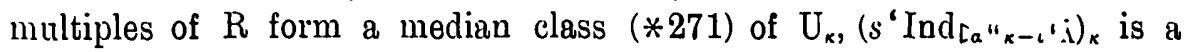
relation which holds between the member of $\kappa$ containing $S$ and $R$, if we understand the use of $\kappa$ as a subscript in the sense defined in $* 356-01$. With the aid of $* 351-11, * 336-44, * 270-4, * 271-15$, we arrive at the conclusion,

$$
\begin{aligned}
& \vdash: \kappa \epsilon \text { FM sr init. }\left(C^{\prime} R_{\kappa}\right) \operatorname{med} U_{\kappa} \cdot \mathrm{Cnv}^{\prime} \dot{s}^{\prime}{ }^{\prime} \kappa_{\partial} \epsilon \text { semi Ded. }
\end{aligned}
$$

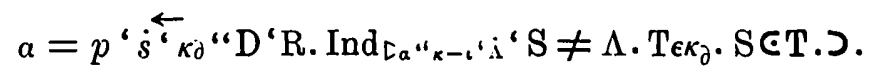

$$
\begin{aligned}
& \mathrm{T}\left(\dot{s}^{\prime} \operatorname{Ind}_{[a}{ }^{\prime \prime}{ }_{k-1}{ }^{\prime} \dot{\alpha} ' S\right) \mathrm{R}
\end{aligned}
$$

Now, if it is true that by repeating any vector belonging to $\kappa_{\partial}$ a suffcient number of times, we can get beyond any given vector belonging to

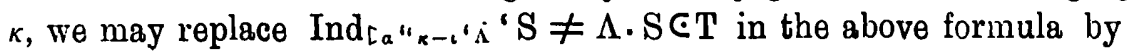

$$
\mathrm{S}=\mathrm{T}[\alpha \neq \Lambda,
$$

as follows readily enough from $* 352-72, * 336-64$. That this repetition is possible is a consequence of $* 337-13$ and $* 336-01-011$. We 
thus obtain the following theorem:

$$
\begin{aligned}
& \left.\vdash \text { : } \kappa \epsilon F M \text { sr init. (C' } R_{\kappa}\right) \text { med } U_{\kappa} \text {. Cnv's ' } \kappa_{\partial} \in \text { semi Ded. }
\end{aligned}
$$

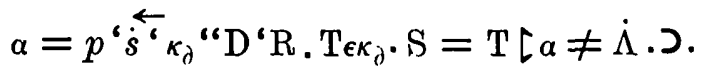

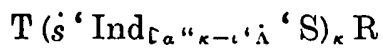

Now, we have seen that $\mathrm{T}\left[a \neq \dot{\Lambda} . \equiv . \mathrm{RU}_{\kappa} \mathrm{T}\right.$, under the above conditions. I wish to show that, given any vector belonging to $\kappa$, there is some member of $\overleftarrow{U}_{k}$ ' $R$, say $Q$, such that $P \in P_{0}{ }^{\prime} Q$. We prove this as follows: by $* 337-13$ and $* 336-01-011$, there is some power of $R$, say $R^{\nu}$, which bears the relation $\mathrm{U}_{\kappa}$ to $\mathrm{P}$. $\mathrm{By} * 337-27$, there is a member of $\kappa$, say $M$, such that $M^{\nu}=P$. This $M$ is the $Q$ we want, since by *336, if $\kappa$ is a serial family, and $\nu$ a positive integer, $\mathrm{RU}_{\kappa} \mathrm{S}$ is equivalent to $\mathrm{R}^{\nu} \mathrm{U}_{\kappa} \mathrm{S}^{\nu}$. Hence, if $\mathrm{P} \epsilon \kappa_{\partial}$, it follows from $* 356-33$ and a little elementary arithmetic that the class $\overrightarrow{\operatorname{In}} x_{x, a}$ ' $P$ contains just two membersthe real number which, when applied, relates $\mathrm{P}$ to $\mathrm{R}$-and that if $\mathrm{P} \sim \epsilon \kappa_{\gamma}, \mathrm{Inx}_{\kappa, u}$ ' $\mathrm{P}$ contains $\Lambda$ alone, Meas $\mathrm{s}_{\kappa, \alpha}$ ' $x$ will then be the upper limit or maximum of the measures in terms of $\mathrm{R}$ of the vectors leading up to $x$. Since these measures, by $* 356-63$, are themselves each the limit or maxima of the class applied rational numbers which the rational multiples of $\mathrm{R}$ less than some vector leading up to $x$ bear to $\mathrm{R}$, Meas $\mathrm{s}_{\mathrm{r}, \mathrm{a}}$ ' $x$ may easily be shown to be the class of all the ratios which vectors which con. nect members of $\overleftarrow{s^{6}} \kappa_{j}$ ' $x$ bear to $\mathrm{R}$, and consequently to be a real num. ber (cf. *336-41,*352-72), such that, when we apply it to $\kappa$, the vector leading from $x$ to init ' $\kappa$ bears it to $R$. In symbols, we have

$$
\begin{aligned}
& \vdash: \mathrm{Hp}(45) \cdot x \epsilon s \text { ' C “ } \kappa . \supset \text {. } \\
& {\left[(\kappa 1 \bar{c})^{\prime}\left(x \downarrow \text { init }{ }^{\prime} \kappa\right)\right]\left(\dot{s}^{\prime}{ }^{\prime} \mathrm{Meas}_{\kappa_{1} a}{ }^{\prime} x\right)_{\kappa} \mathrm{R}}
\end{aligned}
$$

Now, it follows from the assumption $\left(C\right.$ ' $\left.R_{k}\right)$ med $U_{k}$ that every member' of $\kappa$ has a real measure in terms of R. Since $\dot{s}^{\prime} \kappa_{\partial}$ is serial, and since, by

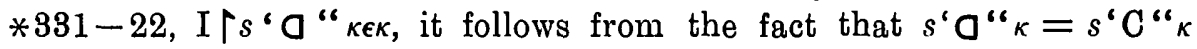
in every vector family that any two members of $s{ }^{\circ} \mathrm{C}$ " $K$ are connected by a member of $\kappa$. Hence we may deduce from $* 356-26-54$ that if $\mu$ is positive, Dist $\kappa_{\kappa, a}{ }^{\prime} \mu \epsilon \kappa_{\partial}$ if $\mu$ is zero, Dist ${ }_{\kappa, a}{ }^{\prime} \mu=I \uparrow s^{\prime} a$ " ${ }^{\prime} \kappa$, while if $\mu$ is negative, Dist ${ }_{\kappa, a}$ ' $\mu \epsilon \mathrm{Cnv}^{\prime}{ }^{\prime} \kappa_{\lambda}$. From this, by $* 356-26$, and the fact that vectors standing in the relation $x_{\kappa}$ to $\mathrm{R}$ always exist if $\kappa$ is initial, serial, submultipliable, and semi-Dedekindian, and $x$ is a non-negative relational 
real number, we may deduce the conclusion

$$
\vdash: \mathrm{Hp}(45) \cdot \supset \cdot \operatorname{Reg}_{\alpha, a}={ }_{\kappa} \cup \mathrm{Cnv} \text { " } \kappa
$$

Another easily proved theorem is

$$
\text { ト : } \mathrm{Hp}(45) \cdot \text {. . } \mathrm{P}\left(s^{\prime} \overline{\mathrm{Dist}}_{\kappa, a} \cdot \mathrm{P}\right)_{\kappa} \mathrm{R}
$$

By (30) and $* 356-33-26$, if $\mu$ is a positive or zero real number, and $\mathrm{P}\left(\mu_{\kappa, a}[\kappa) \mathrm{Q}\right.$, then we have, in the sense of $* 356, \mathrm{P}\left(\dot{s}^{c} \mu\right)_{\kappa} Q$. The converse of this is easily proved by the same theorems, since $P$ and $(?$ always bear some applied real number to $R$ : that is, we have

$$
\vdash: \operatorname{Hp}(45) \cdot \mu \epsilon C^{\prime} \theta^{\prime} . \supset \cdot \mu_{\kappa, a}\left[\kappa=\left(\dot{s}^{`} \mu\right)_{\kappa}\right.
$$

This is the theorem we set out to prove, and it establishes the fact that in an initial, serial, semi-Dedekindian family, if the rational multiples of a given vector form a median class of the series of vectors, the system of measurement defined in the Principia gives substantially the $\operatorname{sam} \theta$ results as the ssstem defined in this paper, if, to put it crudely, we take this given vector whose rational multiples form a median class of the series of vectors as a unit, by making the class of all the things which follow every member of its domain in the series generated by the vectors the $a$ of our previous work.*

5. In conclusion, let us consider what bearing all this work of ours can have on experimental psychology. One of the great defects under which the latter science at present labours is its propensity to try to answer questions without first trying to find out just what they ask. The experimental investigation of Weber's law is a case in point: what most experimenters do take for granted before they begin their experiments is infinitely more important and interesting than any results to which their experiments lead. One of these unconscious assumptions is that sensations or sensation-intervals can be measured, and that this process of measurement can be carried out in one way only. As a result, each new experimenter would seem to have devoted his whole energies to the invention of a method of procedure logically irrelevant to everything that had gone before: one man asks his subject to state when two intervals between sensations of a given kind appear different; another bases his

- It will be noticed that in such a vector family, the series of vectors, arranged in order of magnitude, is of the form $(\overline{+}+\bar{i})$ ?. 
whole work on an experiment where the observer's only problem is to divide a given colour-interval into two equal parts, and so on indefinitely, while even where the experiments are exactly alike, no two people choose quite the same method for working up their results. Now, if we make a large number of comparisons of sensation-intervals of a given sort with reference merely to whether one seems larger than another, the methods of measurement given in this paper indicate perfectly unambiguous ways of working up the results so as to obtain some quantitative law such as that of Weber, without introducing such bits of mathematical stupidity as treating a "just noticeable difference" as an "infinitesimal," and have the further merit of always indicating some tangible mathematical conclusion, no matter what the outcome of the comparisons may be.

Massachusetts Institute of Technology. 